\title{
Maximizing the descent statistic
}

\author{
RichaRd EHRENBORG AND SwAPNEEL MAHAJAN
}

\section{Contents}

1 Introduction

2 The generalized boustrophedon transform

3 The partial order on lists

4 Stronger implications

5 On lists containing palindromes

6 On the balancing of lists

7 An application to inequalities for the Euler numbers

\begin{abstract}
For a subset $S$, let the descent statistic $\beta(S)$ be the number of permutations that have descent set $S$. We study inequalities between the descent statistics of subsets. Each subset (and its complement) is encoded by a list containing the lengths of the runs. We define two preorders that compare different lists based on the descent statistic. Using these preorders, we obtain a complete order on lists of the form $\left(k^{i}, P, k^{n-i}\right)$, where $P$ is a palindrome, whose first entry is larger than $k$. We prove a conjecture due to Gessel, which determines the list that maximizes the descent statistic, among lists of a given size and given length. We also have a generalization of the boustrophedon transform of Millar, Sloane and Young.
\end{abstract}




\section{Introduction}

Let $\mathcal{S}_{n}$ be the symmetric group on $n$ elements. For a permutation $\pi=\pi_{1}, \ldots, \pi_{n+1}$ in the symmetric group on $n+1$ elements, $\mathcal{S}_{n+1}$, define the descent set of $\pi$ to be the set

$$
\operatorname{des}(\pi)=\left\{i \quad \pi_{i}>\pi_{i+1}\right\} .
$$

Observe that $\operatorname{des}(\pi)$ is a subset of $\{1, \ldots, n\}$.

Definition 1.1 For a subset $S$ of $\{1, \ldots, n\}$, define the descent statistic $\beta(S)$ to be the number of permutations in the symmetric group $\mathcal{S}_{n+1}$ that have descent set $S$.

A central problem is to study how different subsets compare on the basis of the descent statistic. The approach that we take in this paper is to try and understand this statistic by restricting to certain families of subsets.

The problem of maximizing $\beta(S)$, where $S$ ranges over all subsets of the set $\{1, \ldots, n\}$, is answered by the following result:

Theorem 1.2 (Niven, de Bruijn) The two subsets of $\{1, \ldots, n\}$ that maximize the descent statistic $\beta(S)$ are

$$
\{1,3,5, \ldots\} \cap\{1, \ldots, n\} \quad \text { and } \quad\{2,4,6, \ldots\} \cap\{1, \ldots, n\} .
$$

This theorem was independently proved by Niven [9] and de Bruijn [2]. The connection between this problem and the cd-index was pointed out by Stanley and can be found in [10]. A short and elegant proof of this theorem using the cd-index appears in [4]. Another proof was given by Viennot [14]. In this paper, we are generalizing the method used by Viennot.

Sagan, Yeh and Ziegler [11] considered the problem of maximizing the Möbius function over certain classes of subposets of the Boolean algebra. Two of these problems can be stated in terms of permutations: Maximize $\beta(S)$, where $S$ is a subset of the form $\{1, \ldots, i\}$, and maximize $\beta(S)$, where $S$ is a subset of the form $\{j, \ldots, k\}$. Their results, loosely stated, say that in the first problem, the maximum is reached when $i$ is about $n / 2$. In the second problem, the maximum is reached when $j$ is about $n / 3$ and $k$ is about $2 n / 3$.

A run in a subset $S$ of the set $\{1, \ldots, n\}$ is a set of consecutive integers of maximal length such that all of them are in the set $S$, or all are not in the set $S$. For instance, the subset $\{1,2,6,7,8\}$ of $\{1, \ldots, 9\}$ has four runs, namely $\{1,2\},\{3,4,5\},\{6,7,8\}$ and $\{9\}$. In general, a subset $S$ of the set $\{1, \ldots, n\}$, up to complement, can be specified as a list, using the lengths of its runs. The set $\{1,2,6,7,8\}$ and its complement $\{3,4,5,9\}$, are described by the list $(2,3,3,1)$. Hence, the results mentioned in the previous paragraph can be stated as follows: The maximum of $\beta(S)$, over all subsets with 2 (or 3) runs, is reached when each run has approximately the same length. 
Gessel conjectured that, when maximizing $\beta(S)$ over all subsets with $k$ runs, maximum is reached when each run is about $n / k$ long. He also conjectured the subsets that attained the maximum. In this paper, we prove his conjecture; see Theorem 6.1.

We also study another class of lists. Let $P=(p, q, \ldots, q, p)$ be a palindrome, that is, it reads the same forwards and backwards. Also, let $k$ be a positive integer less than $p$. Consider lists of the form

$$
\left(k^{i}, P, k^{j}\right)=(\underbrace{k, \ldots, k}_{i}, p, q, \ldots, q, p, \underbrace{k, \ldots, k}_{j})
$$

where $i+j=n$. We determine the complete order among the values $\beta\left(k^{i}, P, k^{j}\right)$ for a given $k, P$ and $n$. Surprisingly, the maximum is reached when $i=1$ and $j=n-1$.

In Section 2, we generalize the boustrophedon transform of Millar, Sloane and Young [8]. Application of this transform to the sequence $(1,0,0,0, \ldots)$ gives us an effective way to compute the values $\beta(S)$.

In Section 3, we introduce two preorders on the set of lists. The weaker preorder is defined by $L \geq M$ if $\beta(L) \geq \beta(M)$, that is, it compares the descent statistics of the two lists. The important result we prove for this preorder is Theorem 6.7. It says that balancing the entries in a list increases the descent statistic. This is the main step in proving the Gessel conjecture.

The second preorder $\succeq$ that we define, makes a more refined, and hence stronger, comparison between two lists. It compares the last row of entries in the boustrophedon transform applied to the sequence $(1,0,0,0, \ldots)$. We denote this array with $R(L)$ for the list $L$. Moreover, this preorder is proved to be a partial order. This partial order is preserved under concatenation on the right; see Corollary 3.8. This gives us a method to build larger relations from smaller relations.

The most important result in building relations is Proposition 4.2. This proposition gives a definite way on how two arrays $R(L)$ and $R(M)$ compare. The two lemmas in Section 4 use this result to give conditions on how the weak preorder implies the stronger partial order. These lemmas are extensively used in Sections 5 and 6.

We end this paper with an application to Euler numbers in Section 7. Using an observation due to MacMahon, we obtain all inequalities of the form $\left(\begin{array}{l}n \\ a\end{array}\right) \cdot E_{a} \cdot E_{b}>\left(\begin{array}{l}n \\ c\end{array}\right) \cdot E_{c} \cdot E_{d}$, where $n=a+b=c+d$.

The descent statistic $\beta(S)$ also appears in the theory of partially ordered sets. Let $P$ be a graded partially ordered set (poset) of rank $n+1$. For $S$ a subset of $\{1, \ldots, n\}$, let $f_{S}$ be the number of chains (flags) that has elements on the ranks in $S$. These $2^{n}$ numbers constitute the flag $f$-vector of the poset $P$. The flag $h$-vector is defined by the relation $h_{S}=\sum_{T \subseteq S}(-1)^{|S-T|} \cdot f_{T}$. For the case when the poset is the Boolean algebra $B_{n+1}$ on $n+1$ elements, that is, the face lattice of the $n$-dimensional simplex, we have $h_{S}=\beta(S)$. This is straightforward to prove usings $R$-labelings; see [1, 12].

We make a few short observations about the descent statistics: The descent statistic $\beta(S)$ is preserved under two symmetries. Firstly, $\beta(\bar{S})=\beta(S)$, where $\bar{S}$ denotes the complement of $S$, that is, $\bar{S}=\{1, \ldots, n\}-S$. Secondly, $\beta(n+1-S)=\beta(S)$, where $n+1-S=\{n+1-s: s \in S\}$. Also note that $\beta(\emptyset)=1$. The $n$th Euler number $E_{n}$ is the number of permutations in the symmetric group $\mathcal{S}_{n}$ which are alternating, that is, $E_{n}=\beta(\{2,4, \ldots\} \cap\{1, \ldots, n-1\})$, or equivalently, $E_{n}=$ 


$$
\begin{aligned}
& \underline{2} \\
& \underline{3} \rightarrow 5 \\
& 8 \leftarrow 5 \leftarrow \underline{0} \\
& 19 \leftarrow 11 \leftarrow 6 \leftarrow \underline{6} \\
& \underline{7} \rightarrow 26 \rightarrow 37 \rightarrow 43 \rightarrow 49
\end{aligned}
$$

Figure 1: The boustrophedon of the sequence $\mathbf{a}=(2,3,0,6,7, \ldots)$ with respect to the set $S=$ $\{1,4, \ldots\}$.

$\beta(\{1,3, \ldots\} \cap\{1, \ldots, n-1\})$. The exponential generating function for the Euler numbers is given by

$$
\sum_{n \geq 0} E_{n} \cdot \frac{x^{n}}{n !}=\tan (x)+\sec (x)
$$

\section{The generalized boustrophedon transform}

We generalize the boustrophedon transform of Millar, Sloane and Young [8]. Let $S$ be a subset of the positive integers $\mathbb{P}$ and let $\mathbf{a}=\left(a_{0}, a_{1}, \ldots\right)$ be a sequence of numbers. We define an infinite triangular array $t_{n, i}(S)=t_{n, i}$, where $0 \leq i \leq n$, by the recursion: The initial condition is $t_{n, 0}=a_{n}$ and the recursive step is:

$$
t_{n, i}= \begin{cases}t_{n, i-1}+t_{n-1, i-1} & \text { if } n-1, n \in S \text { or } n-1, n \notin S, \\ t_{n, i-1}+t_{n-1, n-i} & \text { otherwise, }\end{cases}
$$

for $1 \leq j \leq i$. Let $b_{n}$ denote the entry $t_{n, n}$. We call the sequence $\mathbf{b}=\left(b_{0}, b_{1}, \ldots\right)$ the boustrophedon transform of the sequence $\mathbf{a}=\left(a_{0}, a_{1}, \ldots\right)$ with respect to the set $S$. Note that the dependence of $t_{i, j}$ on the sequence a and the set $S$ is suppressed in our notation. We may visualize the computation in terms of a triangle; see Figure 1. In the $i$ th row, we add going to the right if $i$ is in the set $S$, otherwise we add to the left. But observe that the indexing of the entries in the triangle does not correspond directly to their positions; see Figure 2.

For $S$ a set of positive integers and $n$ and $j$ non-negative integers, define $S_{n, j}$ to be the set $\{s \in\{1, \ldots, n-j\}: s+j \in S\}$. Observe that $S_{n, 0}$ is the set $S \cap\{1, \ldots, n\}$. Also, let $\mathbf{e}_{j}$ be the $j$ th unit sequence, that is, $\mathbf{e}_{j}=(\underbrace{0, \ldots, 0}_{j}, 1,0, \ldots)$.

Proposition 2.1 Let $S$ be a subset of the positive integers $\mathbb{P}$. The boustrophedon transform of the sequence $\mathbf{a}=\left(a_{0}, a_{1}, \ldots\right)$ with respect to the set $S$ is given by:

$$
b_{n}=\sum_{j=0}^{n}\left(\begin{array}{l}
n \\
j
\end{array}\right) \cdot \beta\left(S_{n-1, j}\right) \cdot a_{j}
$$

We postpone the proof of this proposition until the end of this section. 


$$
\begin{aligned}
& \begin{array}{c}
\frac{t_{1,0}}{\leftarrow} \stackrel{t_{0,0}}{\rightarrow} t_{1,1} \\
t_{2,2} \leftarrow t_{2,1} \leftarrow t_{2,0}
\end{array} \\
& t_{3,3} \leftarrow t_{3,2} \leftarrow t_{3,1} \leftarrow t_{3,0} \\
& \underline{t_{4,0}} \rightarrow t_{4,1} \rightarrow t_{4,2} \rightarrow t_{4,3} \quad \bar{\rightarrow} t_{4,4}
\end{aligned}
$$

Figure 2: The pattern of the entries of the boustrophedon with respect to the set $S=\{1,4, \ldots\}$.

When the subset $S$ is one of the alternating sets, that is, $S=\{1,3,5, \ldots\}$ or $S=\{2,4,6, \ldots\}$, we obtain the boustrophedon transform of Millar, Sloane and Young. Then the identity in Proposition 2.1 specializes to

$$
b_{n}=\sum_{j=0}^{n}\left(\begin{array}{l}
n \\
j
\end{array}\right) \cdot E_{n-j} \cdot a_{j},
$$

which in terms of generating functions can be written as:

$$
\sum_{n \geq 0} b_{n} \cdot \frac{x^{n}}{n !}=(\tan (x)+\sec (x)) \cdot \sum_{n \geq 0} a_{n} \cdot \frac{x^{n}}{n !}
$$

see Theorem 1 in [8].

The boustrophedon transform, when applied to the sequence $\mathbf{e}_{0}=(1,0, \ldots)$, generates the descent statistic of the set $S$. In addition, we interpret the rows of the transform as a refined descent statistic; see Definition 2.2 and Lemma 2.4. This refined descent statistic and the original statistic are closely related and are useful in later applications. We will explore this connection in the following sections.

Definition 2.2 Let $S$ be a subset of $\{1, \ldots, n\}$. We define $\beta_{i}(S)$, where $1 \leq i \leq n+1$, by two cases: If $n \notin S$ then $\beta_{i}(S)$ is the number of permutations in the symmetric group $\mathcal{S}_{n+1}$ with descent set $S$ that end in the letter $i$. If $n \in S$ then $\beta_{i}(S)$ is the number of permutations in $\mathcal{S}_{n+1}$ with descent set $S$ that end in the letter $n-i+2$.

The numbers $\beta_{i}(S)$ are a refinement of the number $\beta(S)$. In particular, $\beta(S)=\sum_{i=1}^{n+1} \beta_{i}(S)$. Also $\beta(S \cap\{1, \ldots, n-1\})=\beta_{n+1}(S)$. Analogous to the statement $\beta(S)=\beta(\bar{S})$, we have,

Lemma 2.3 For $S$ a subset of $\{1, \ldots, n\}$ and for $1 \leq i \leq n+1$, we have $\beta_{i}(S)=\beta_{i}(\bar{S})$.

We now have the following lemma, which is also due to Viennot [14, Proposition 2].

Lemma 2.4 Consider the boustrophedon transform of the sequence $\mathbf{e}_{0}=(1,0,0, \ldots)$. Then $t_{n, i}(S)$ is equal to the number of permutations in the symmetric group $\mathcal{S}_{n+1}$ on $n+1$ elements with descent set $S \cap\{1, \ldots, n\}$ and ending in $i+1$ if $n \notin S$, otherwise ending in $n-i+1$. In particular, we have $t_{n, n}(S)=\beta(S \cap\{1, \ldots, n-1\})$. 
It is straightforward to prove Lemma 2.4 directly. We, however, have chosen a different path, since we are interested in proving Proposition 2.1. The reader, who is interested in the later sections, may skip directly to Section 3 without losing anything essential.

Lemma 2.4 is a special case of Lemma 2.5. We now work towards the proof of Proposition 2.1 and Lemma 2.5. The key step in it is to understand the transform of the sequence $\mathbf{e}_{j}$. This analysis requires the notion of partial permutations.

Recall that a permutation is an array of length $n$, where the entries are from the set $\{1,2, \ldots, n\}$, and each element appears exactly once. A partial permutation on the set $\{1,2, \ldots, n\}$ of length $k$ is an array of length $k$, where entries are from the set $\{1,2, \ldots, n\}$, and each element appears at most once. Similarly, we define the descent set of a partial permutation $\pi=\pi_{1}, \ldots, \pi_{k}$ to be the set

$$
\operatorname{des}(\pi)=\left\{i: \pi_{i}>\pi_{i+1}\right\} .
$$

Observe that $\operatorname{des}(\pi)$ is a subset of $\{1, \ldots, k-1\}$.

Let $S$ be a subset of $\{1, \ldots, k-1\}$. Note that the number of partial permutations on the set $\{1, \ldots, n\}$ of length $k$ with descent set $S$ is given by $\left(\begin{array}{l}n \\ k\end{array}\right) \cdot \beta(S)$.

Lemma 2.5 Consider the boustrophedon of the sequence $\mathbf{e}_{j}$, where $j$ is a non-negative integer. Then $t_{n, i}(S)$ is equal to the number of partial permutations on the set $\{1, \ldots, n+1\}$ of length $n-j+1$ with descent set $S_{n, j}$ and ending in $i+1$ if $n \notin S$, otherwise ending in $n-i+1$.

Proof: Let $\beta_{n, i}$ denote the number of partial permutations described in the statement of the lemma. We suppress $j$ in our notation. By setting $i=0$, we see that $\beta_{n, 0}=1$ if $n=j$ and zero otherwise.

To obtain a recursion for $\beta_{n, i}$, one has to consider four cases, depending on whether $n-1$ belongs to $S$, and whether $n$ belongs to $S$. We first consider the case when $n-1, n \notin S$. Let $P_{n, i}$ be the set of partial permutations of length $n-j+1$ with descent set $S_{n, j}$ and ending in $i+1$. Note that $\beta_{n, i}$ is the cardinality of $P_{n, i}$. Since $n-1, n \notin S$ a partial permutation in $P_{n, i}$ satisfies $\pi_{n-j-1}<\pi_{n-j}<$ $\pi_{n-j+1}=i+1$. Hence, we can decompose the set $P_{n, i}$ into two disjoint subsets:

$$
\begin{aligned}
& A_{1}=\left\{\pi \in P_{n, i}: \pi_{n-j}<i\right\}, \\
& A_{2}=\left\{\pi \in P_{n, i}: \pi_{n-j}=i\right\} .
\end{aligned}
$$

The set $A_{2}$ is in bijection with the set $P_{n-1, i-1}$ by removing the last element of the partial permutation and relabeling the elements $i+2, \ldots, n+1$ by $i+1, \ldots, n$. The set $A_{1}$ is in bijection with the set $P_{n, i-1}$ by relabeling $i+1$ by $i$, and relabeling $i$ (if it occurs) by $i+1$. Hence, we obtain

$$
\beta_{n, i}=\beta_{n, i-1}+\beta_{n-1, i-1} \quad \text { if } n-1, n \notin S .
$$

The other three cases can be dealt with similarly and we get the recursion:

$$
\beta_{n, i}= \begin{cases}\beta_{n, i-1}+\beta_{n-1, i-1} & \text { if } n-1, n \in S \text { or } n-1, n \notin S, \\ \beta_{n, i-1}+\beta_{n-1, n-i} & \text { otherwise. }\end{cases}
$$


Observe that $\beta_{n, i}$ satisfies the same recursion and initial conditions as $t_{n, i}$. Hence $\beta_{n, i}=t_{n, i}$ and the lemma is established.

By substituting $i=n$ in Lemma 2.5, we see that $t_{n, n}(S)$ is the number of partial permutations on the set $\{1, \ldots, n\}$ of length $n-j$ with descent set $S_{n-1, j}$. Hence, we obtain the following corollary:

Corollary 2.6 With the same notation and condition as in Lemma 2.5, we have

$$
t_{n, n}(S)=\left(\begin{array}{l}
n \\
j
\end{array}\right) \cdot \beta\left(S_{n-1, j}\right) .
$$

We can now prove the main proposition of this section.

Proof of Proposition 2.1: Since the boustrophedon transform is a linear function, it is enough to prove it for the $j$ th unit sequence $\mathbf{e}_{j}$. But the case $\mathbf{a}=\mathbf{e}_{j}$ is a straightforward consequence of Corollary 2.6.

\section{$3 \quad$ The partial order on lists}

In the remainder of this paper, we will be using lists to denote subsets, as were described in the introduction. Observe that the list $(2,3,3,1)$ corresponds to two subsets, namely $\{1,2,6,7,8\}$ and its complement $\{3,4,5,9\}$. This is unambiguous for us since $\beta(S)=\beta(\bar{S})$ and more generally $\beta_{i}(S)=$ $\beta_{i}(\bar{S})$. Hence, we can write $\beta(L)$ and $\beta_{i}(L)$ for a list $L$.

We will abuse the notation and let $(K, L)$ denote the concatenation of the two lists $K$ and $L$. For instance, $(K, L, m, n, M)$ denotes the concatenation of the lists $K, L,(m, n)$ and $M$, and $\beta(M, m)$ is the descent statistic applied to the list $(M, m)$. The reverse of a list $L$ is denoted by $L^{*}$. Observe that $\beta(L)=\beta\left(L^{*}\right)$. A list $P$ that satisfies $P=P^{*}$ is called a palindrome. The size of a list is the sum of its entries. Let $\epsilon$ denote the empty list. Moreover, we let the superindex denote repetition, that is, $\left(k^{a}\right)$ is the list $(\underbrace{k, \ldots, k}_{a})$.

For a list $L$, let $L-1$ denote the list with one subtracted from the last entry. That is, for $L=(M, m)$, we have $L-1=(M, m-1)$ if $m \geq 2$ and $L-1=M$ if $m=1$.

In general, our lists contains positive integers. But in order to be able to state certain results, we will allow entries of a list to be zero. This does not change the underlying set, for instance the list $(L, 0, M)$ corresponds to the same subsets as the list $(L, M)$. The two lists $(L, 0, M)$ and $(L, M)$ will behave exactly the same with the descent statistics, that is, $\beta_{i}(L, 0, M)=\beta_{i}(L, M)$. Also, let the length of a list be the number of non-zero entries. 


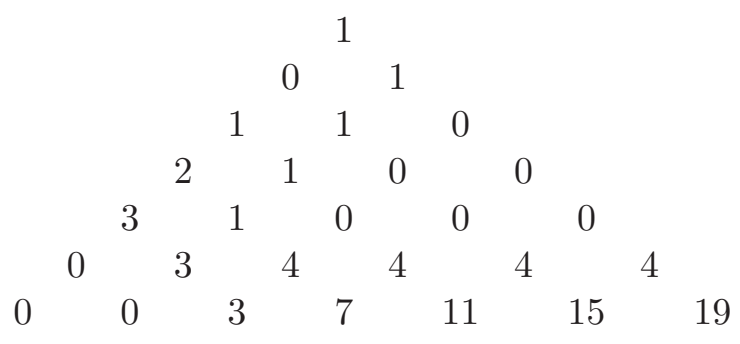

Figure 3: Calculation of $R(1,3,2)=(0,0,3,7,11,15,19)$ and $\beta(1,3,2)=0+0+3+7+11+15+19=55$.

Define a linear map from $\mathbb{R}^{n}$ to $\mathbb{R}^{n+1}$ by $\Sigma(v)=\left(0, v_{1}, v_{1}+v_{2}, \ldots, v_{1}+\cdots+v_{n}\right)$ for the vector $v=\left(v_{1}, \ldots, v_{n}\right)$. Also, define the reverse of the vector $v$ to be the vector $v^{*}=\left(v_{n}, \ldots, v_{1}\right)$. Define a partial order on $\mathbb{R}^{n}$ by $v \geq w$ if $v$ is component-wise greater than or equal to $w$, that is, $v_{i} \geq w_{i}$ for all indices $i$. Moreover, we say that $v>w$ if $v \geq w$ and $v \neq w$. Observe that $*$ and $\Sigma$ are injective and preserve this partial order, that is, $v>w$ implies $v^{*}>w^{*}$ and $\Sigma(v)>\Sigma(w)$.

For $L$ a list of size $n$, define the array $R(L)$ to be the vector

$$
R(L)=\left(\beta_{1}(L), \ldots, \beta_{n+1}(L)\right)
$$

By Lemma 2.4, the array $R(L)$ can be computed by the boustrophedon transform applied to the sequence $\mathbf{e}_{0}=(1,0, \ldots)$; see Figure 3 . For instance, for the two-element list $(m, n)$, we have $\beta(m, n)=$ $\left(\begin{array}{c}m+n \\ m\end{array}\right)$ and

$$
R(m, n)=(\underbrace{0, \ldots, 0}_{n},\left(\begin{array}{c}
n-1 \\
n-1
\end{array}\right), \ldots,\left(\begin{array}{c}
m+n-1 \\
n-1
\end{array}\right)) .
$$

This follows, since the Pascal's triangle appears in the calculation of $R(m, n)$.

Observe that the entries of $R(L)$ are weakly increasing, with zero as the first entry and $\beta(L-1)$ as the last entry. In the previous section, we had described the recursion on the triangular pattern in terms of its individual elements. However, it is more easily described in terms of the linear operations $\Sigma$ and $*$ on its rows, which we have denoted by $R(L)$. Hence we obtain:

Lemma 3.1 For a list $L$, we have $R(L, 1)=\Sigma\left(R(L)^{*}\right)$ and $R(L, m+1)=\Sigma(R(L, m))$.

Corollary 3.2 Let $m$ be a positive integer and $M$ be any list. Then the first $m$ entries of $R(M, m)$ are zero and the entry in the $(m+1)$ st position is given by $\beta(M-1)$. In particular, it is non-zero.

Lemma 3.3 Let $L$ and $M$ be two lists of the same size. Then the inequality $R(L)>R(M)$ implies $\beta(L)>\beta(M)$ and $\beta(L-1) \geq \beta(M-1)$.

Proof: The inequality is true, since $\beta(L)$ is the sum of the entries of $R(L)$ and $\beta(L-1)$ is the last entry of $R(L)$. 
We are primarily interested in comparing two lists depending on their $\beta$-values. We define two different preorders, namely $\geq$ and $\succeq$ on the set of lists of a given size by:

Definition 3.4 Let $L$ and $M$ be two lists of the same size. We define the two preorder relations $L \geq M$ if $\beta(L) \geq \beta(M)$ and $L \succeq M$ if $R(L) \geq R(M)$. We say that $L>M$ if $\beta(L)>\beta(M)$. Similarly, $L \succ M$ if $L \succeq M$ and $L \neq M$.

Note that the preorder $\geq$ does not satisfy the antisymmetry axiom and hence is not a partial order, for instance $\beta(L)=\beta\left(L^{*}\right)$.

Lemma 3.5 The preorder $\succeq$ is a partial order, that is, the relation $\succeq$ is antisymmetric.

Proof: Assume that we have two lists $M$ and $N$ such that $M \succeq N$ and $N \succeq M$. That is, $R(M)=$ $R(N)$. Let $m$ be the number of zeros in the beginning of the array $R(M)$. By Corollary 3.2, the last entry of the list $M$ is $m$. Hence, we may write $M=\left(M^{\prime}, m\right)$. Similarly, we obtain $N=\left(N^{\prime}, m\right)$. Since the linear map $\Sigma$ is injective, the linear map $v \longmapsto \Sigma^{m}\left(v^{*}\right)$ is injective and we have $R\left(M^{\prime}\right)=R\left(N^{\prime}\right)$. By repeating this argument, we obtain that the two lists $M$ and $N$ are equal.

Lemma 3.3 can be restated in terms of our new definitions by:

Lemma 3.6 Let $L$ and $M$ be two non-empty lists of the same size. Then $L \succ M$ implies $L>M$ and $(L-1) \geq(M-1)$.

Lemma 3.7 If $L$ and $M$ are two non-empty lists of the same size, then $L \succ M$ implies $(L, 1) \succ(M, 1)$. If $(L, l)$ and $(M, m)$ are two lists of the same size, then $(L, l) \succ(M, m)$ implies $(L, l+1) \succ(M, m+1)$.

Proof: By Lemma 3.1, we have $R(L, 1)=\Sigma\left(R(L)^{*}\right)$. Since the two linear maps $\Sigma$ and $*$ preserve the inequality $>$ on vectors, the first statement follows. The second statement is proved similarly.

This lemma has the following consequence:

Corollary 3.8 Let $L$ and $M$ be two non-empty lists of the same size. Then $L \succeq M$ implies $(L, N) \succeq$ $(M, N)$ for any list $N$.

The partial order $\succeq$, however, is not preserved under concatenation from the left. For instance, $(1,2,1) \succ(2,1,1)$ but $(1,1,2,1) \nsucceq(1,2,1,1)$.

Lemma 3.9 For a list $M$ and two positive integers $m$ and $n$, we have $(M, m, n, N) \succ(M, m+n, N)$. 
Proof: In view of Corollary 3.8 and Lemma 3.7, it is enough to consider the case when $N$ is the empty list and $n$ is equal to 1 . Since $R(M, m)$ has increasing entries, the sum of the first $i$ entries is less than or equal to the sum of the last $i$ entries. In other words, we have $\Sigma(R(M, m)) \leq \Sigma\left(R(M, m)^{*}\right)$. Compare the second entry in this inequality. It is zero in the first term, but positive in the second. Hence, we have the strict inequality $\Sigma(R(M, m))<\Sigma\left(R(M, m)^{*}\right)$, which is the desired result.

Lemma 3.9 generalizes Lemma 2 in [14] and the alternating property in [4]. Repeated application of this lemma gives us the following theorem:

Theorem 3.10 The list $\left(1^{n}\right)=(1, \ldots, 1)$ is the unique maximal element in the partial order $\succeq$ among all lists of size $n$.

Since the list $\left(1^{n}\right)$ corresponds to the two alternating sets, this result implies Theorem 1.2. Viennot's proof of Theorem 1.2 follows the same ideas.

\section{Stronger implications}

In the previous section, we defined a partial order, namely $>$, on the vectors in $\mathbb{R}^{n}$. We now introduce another notion of comparison, which we denote by (+-). Its usefulness will be clear from Proposition 4.2.

Definition 4.1 Let $v$ and $w$ be two vectors in $\mathbb{R}^{n}$. We define the relation $v+-w$ to mean that there exists an index $j$ such that

- for $1 \leq i \leq j$, we have $v_{i} \geq w_{i}$ and at least one of these inequalities is strict,

- for $j+1 \leq i \leq n$, we have $v_{i}<w_{i}$.

The relation $v-+w$ is defined as the reverse relation, that is, $v-+w$ iff $w+-v$. Define the relation $v_{>}^{+-} w$ to mean $v>w$ or $v+-w$. Similarly, define the relation $v_{<}^{-+} w$ to mean $v<w$ or $v-+w$.

Proposition 4.2 Let $L$ and $M$ be two different lists of the same size. Then the arrays $R(L)$ and $R(M)$ compare in one of the four following ways: $R(L)>R(M), R(L)+-R(M), R(L)-+R(M)$ or $R(L)<R(M)$.

Proof: The proof is by induction on $n$, the size of the lists that we compare. The induction base is straightforward, hence we concentrate on the induction step.

For any list $L$, the array $R(L)$ can be inductively constructed from the list (1) by applying the linear maps $v \longmapsto \Sigma(v)$ and $v \longmapsto \Sigma\left(v^{*}\right)$. Hence, to prove the induction step, we need to show that the relations $>,<,+-$ and -+ are closed under these two operations. 
Let $L$ and $M$ be two lists of size $n$. Let $v=R(L)$ and $w=R(M)$. Note that the vectors $v$ and $w$ are non-zero and have weakly increasing entries. By the induction hypothesis and without loss of generality, we may assume that $v>w$ or $v+-w$. The proof breaks up into eight cases. The first four cases are:

- $v>w \Longrightarrow \Sigma(v)>\Sigma(w)$,

- $v>w \Longrightarrow \Sigma\left(v^{*}\right)>\Sigma\left(w^{*}\right)$,

- $u+-v \Longrightarrow \Sigma(u)_{>}^{+-} \Sigma(v)$ and

- $u+-v \Longrightarrow \Sigma\left(u^{*}\right)_{<}^{-+} \Sigma\left(v^{*}\right)$.

These four cases are straightforward. The next four cases are more interesting and we give detailed proofs.

- $v>w \Longrightarrow \Sigma\left(v^{*}\right)>\Sigma(w)$. Since $v$ has increasing entries, the sum of its first $i$ entries is less than or equal to the sum of the last $i$ entries, that is, $\Sigma\left(v^{*}\right)>\Sigma(v)$. Combining this with the fact that $v>w$ implies $\Sigma(v)>\Sigma(w)$, we obtain the desired result.

- $v>w \Longrightarrow \Sigma(v)-+\Sigma\left(w^{*}\right)$. The second entry of $\Sigma(v)$, which is zero, is less than the second entry of $\Sigma\left(w^{*}\right)$, which is non-zero. Also, the last entry of $\Sigma(v)$, by our hypothesis, is greater than that of $\Sigma\left(w^{*}\right)$. Let $i$ be the first integer for which the sum of the first $i$ entries of $v$ is greater than that of $w^{*}$. Since $v$ is increasing, while $w^{*}$ is decreasing, the same is true for all integers greater than $i$. Hence, $\Sigma(v)-+\Sigma\left(w^{*}\right)$.

- $v+-w \Longrightarrow \Sigma\left(v^{*}\right)_{>}^{+-} \Sigma(w)$. The second entry of $\Sigma\left(v^{*}\right)$, which is non-zero, is greater than the second entry of $\Sigma(w)$, which is zero. So, we either have $\Sigma\left(v^{*}\right)>\Sigma(w)$ or there exists an integer $i$ such that the sum of the first $i$ entries of $v^{*}$ is less than that of $w$. In this case, since $w$ is increasing, while $v^{*}$ is decreasing, the same is true for all integers greater than $i$. Hence, $\Sigma\left(v^{*}\right)+-\Sigma(w)$.

- $v+-w \Longrightarrow \Sigma(v)_{<}^{-+} \Sigma\left(w^{*}\right)$. The second entry of $\Sigma(v)$, which is zero, is less than the second entry of $\Sigma\left(w^{*}\right)$, which is non-zero. So, we either have $\Sigma(v)<\Sigma\left(w^{*}\right)$ or there exists an integer $i$ such that the sum of the first $i$ entries of $v$ is greater than that of $w^{*}$. In this case, since $v$ is increasing, while $w^{*}$ is decreasing, the same is true for all integers greater than $i$. Hence, $\Sigma(v)-+\Sigma\left(w^{*}\right)$.

These eight cases complete the induction step and the proposition follows.

Proposition 4.2 has the following two important consequences:

Lemma 4.3 Let $(M, m)$ and $(N, n)$ be two lists of the same size such that $0<m<n$. Then we have $(M, m-1) \geq(N, n-1)$ implies $(M, m) \succ(N, n)$. 
Proof: Since $R(M, m)$ has a smaller string of initial zeroes than $R(N, n)$, we have that the $(m+1)$ st entry in $R(M, m)$ is greater than the $(m+1)$ st entry in $R(N, n)$. Hence by Proposition 4.2 , we have either $R(M, m)>R(N, n)$ or $R(M, m)+-R(N, n)$. Since $\beta(M, m-1) \geq \beta(N, n-1)$, the last entry in $R(M, m)$ is greater than or equal to the last entry in $R(N, n)$. This rules out the second possibility and hence the conclusion of the lemma follows.

Lemma 4.4 Let $M$ and $N$ be two lists of the same size and let $k$ be a non-negative integer. Assume that $(M, k) \geq(N, k)$ and $M-1>N-1$. Then we have $(M, k+1) \succ(N, k+1)$.

Proof: By Corollary 3.2, we have that $R(M, k+1)$ and $R(N, k+1)$ have the same number of initial zeros. But the first non-zero entry in $R(M, k+1)$ is $\beta(M-1)$, which is greater than the first non-zero entry in $R(N, k+1)$. Moreover, the last entry of $R(M, k+1)$ is $\beta(M, k)$ which is greater than or equal to the last entry of $R(N, k+1)$, which is $\beta(N, k)$. Hence by Proposition 4.2 , we obtain the conclusion of the lemma.

In view of Proposition 4.2, we observe that if $R(L)$ and $R(M)$ have the same last entry, then either $L \succ M$ or $M \succ L$. The converse to Lemma 4.3 is true, while the converse to Lemma 4.4 is false.

\section{$5 \quad$ On lists containing palindromes}

In this section, we study a beautiful pattern relating lists that have the special form $\left(k^{a}, P, k^{b}\right)$, where $P$ is a palindrome that starts and ends with the letter $p$, with $p>k$. A special case of this pattern will be used in the next section. Also, the inequalities for the Euler numbers, proved in Section 7, will be a consequence of the results in this section.

We start off with two warm-up lemmas, which are the initial cases of Proposition 5.3. The main result of this section is Theorem 5.4, which is a consequence of Proposition 5.3.

Lemma 5.1 Let $j$ and $k$ be non-negative integers such that $j<k$, and let $P$ be a palindrome that begins (and ends) with an integer greater than $k$. Then we have $(j, k, k, P, k) \succ(j, P, k, k, k)$.

Proof: Assume that $P$ has length at least 2. The case when $P$ has length 1 , that is, $P=(p)$, can be handled similarly. Since $P$ is palindromic, we can write $P=(p, Q, p)$, with $p$ larger than $k$ and $Q$ a palindrome. Since the two lists $(p-1, Q, p, k, k-1)$ and $(k-1, k, p, Q, p-1)$ are reverse of each other, we have $(p-1, Q, p, k, k-1) \geq(k-1, k, p, Q, p-1)$. Applying Lemma 4.3, we get $(p-1, Q, p, k, k) \succ(k-1, k, p, Q, p)$. By Corollary 3.8 , we have $(p-1, Q, p, k, k, j) \succ(k-$ $1, k, p, Q, p, j)$ and hence $(p-1, Q, p, k, k, j)>(k-1, k, p, Q, p, j)$. Now, by reversing the lists, we obtain $(j, k, k, p, Q, p-1)>(j, p, Q, p, k, k-1)$. Also, observe that $(j, k, k, p, Q, p, j) \geq(j, p, Q, p, k, k, j)$, since the two lists are reverse of each other. Applying Lemma 4.4 to the two last inequalities, we have $(j, k, k, p, Q, p, j+1) \succ(j, p, Q, p, k, k, j+1)$, that is, $(j, k, k, P, j+1) \succ(j, P, k, k, j+1)$. Since 
$j+1 \leq k$, we can apply Lemma 3.7 to get the conclusion of the lemma: $(j, k, k, P, k) \succ(j, P, k, k, k)$.

Lemma 5.2 Let $j$ and $k$ be non-negative integers such that $j<k$, and let $P$ be a palindrome that begins (and ends) with an integer greater than $k$. Then we have $(j, k, P, k) \succ(j, P, k, k)$.

The proof is similar to that of the previous lemma and hence we omit it.

Proposition 5.3 Let $P$ be a palindrome that starts and ends with the positive integer $p$. Let $j$ and $k$ be non-negative integers such that $j<k<p$. Then the following four statements hold:

$$
\begin{array}{lll}
A(n):\left(j, k^{2 n+2}, P, k^{2 n+1}\right) & \succ\left(j, k^{2 n}, P, k^{2 n+3}\right) & \text { for } n \geq 0 \\
B(n):\left(j, k^{2 n-1}, P, k^{2 n+2}\right) & \succ\left(j, k^{2 n+1}, P, k^{2 n}\right) & \text { for } n \geq 1 \\
C(n):\left(j, k^{2 n+1}, P, k^{2 n+1}\right) & \succ\left(j, k^{2 n}, P, k^{2 n+2}\right) & \text { for } n \geq 0 \\
D(n):\left(j, k^{2 n-1}, P, k^{2 n+1}\right) & \succ\left(j, k^{2 n}, P, k^{2 n}\right) & \text { for } n \geq 1
\end{array}
$$

Proof: We prove parts $A$ and $B$ of the theorem using a joint induction. The induction is divided in three steps. The first step is the induction basis, which is the statement $A(0)$. The next two steps are the induction steps, which are $A(n)$ implies $B(n+1)$ and $B(n)$ implies $A(n)$.

(i) $A(0)$. This is Lemma 5.1, which completes the induction basis.

(ii) $A(n) \Longrightarrow B(n+1)$. By setting $j=k-1$ in the statement $A(n)$, we obtain

$$
\left(k-1, k^{2 n+2}, P, k^{2 n+1}\right) \succ\left(k-1, k^{2 n}, P, k^{2 n+3}\right) .
$$

By Lemma 3.6, we can append the integer $j$ to the lists to obtain,

$$
\left(k-1, k^{2 n+2}, P, k^{2 n+1}, j\right) \succ\left(k-1, k^{2 n}, P, k^{2 n+3}, j\right) .
$$

By considering the weaker preorder and reversing the lists, we have

$$
\left(j, k^{2 n+1}, P, k^{2 n+2}, k-1\right)>\left(j, k^{2 n+3}, P, k^{2 n}, k-1\right) .
$$

We also have $\left(j, k^{2 n+1}, P, k^{2 n+3}, j\right) \geq\left(j, k^{2 n+3}, P, k^{2 n+1}, j\right)$, since the two lists are reverse of each other. Applying Lemma 4.4 to the last two inequalities, we get

$$
\left(j, k^{2 n+1}, P, k^{2 n+3}, j+1\right) \succ\left(j, k^{2 n+3}, P, k^{2 n+1}, j+1\right) .
$$

Since $j+1 \leq k$, we apply Lemma 3.7 to obtain the statement $B(n+1)$.

(iii) $B(n) \Longrightarrow A(n)$. This case follows the exact outline as the previous case. 




Figure 4: Comparison between the lists $(1,1,2,1)$ and $(2,1,1,1)$.

Hence we conclude that the statements $A$ and $B$ hold. By a similar induction $C$ and $D$ are true.

As an example, consider the statement $A(0)$ with $k=1$ and the palindrome $P=(2)$. That is, we compare the two lists $(1,1,2,1)$ and $(2,1,1,1)$. Their arrays are worked out in Figure 4 . Observe that this implies $\left(1,1,2,1^{n}\right) \succ\left(2,1^{n+2}\right)$ for $n \geq 1$. But for $n=0$, we have $(1,1,2) \nsucc(2,1,1)$. In fact, these arrays satisfy the relation $R(1,1,2)-+R(2,1,1)$. In general, in Proposition 5.3, one cannot decrease the superindex on the right. This observation yields plenty of examples, where the partial order $\succ$ is not preserved under left concatenation; see remark after Corollary 3.8.

Let $T_{n}$ be the set of unordered pairs of non-negative integers $\{a, b\}$ such that $a+b=n$. Define a total order on $T_{n}$ by the following four cases:

- For $n$ odd: For $\{a, b\},\{c, d\} \in T_{n}$, we say that $\{a, b\}>\{c, d\}$ if the odd member in $\{a, b\}$ is smaller than the odd member in $\{c, d\}$.

- For $n$ even:

- For $\{a, b\},\{c, d\} \in T_{n}$, we say that $\{a, b\}>\{c, d\}$ if the smallest odd member in $\{a, b\}$ is smaller than the smallest odd member in $\{c, d\}$.

- For $\{a, b\},\{c, d\} \in T_{n}$, we say that $\{a, b\}>\{c, d\}$ if the smallest even member in $\{a, b\}$ is greater than the smallest even member in $\{c, d\}$.

- For $\{a, b\},\{c, d\} \in T_{n}$, we say that $\{a, b\}>\{c, d\}$ if $a$ and $b$ are odd and $c$ and $d$ are even.

We give two examples of this ordering for $n=8$ and $n=9$ :

$$
\begin{aligned}
& T_{8}:\{1,7\}>\{3,5\}>\{4,4\}>\{2,6\}>\{0,8\}, \\
& T_{9}:\{1,8\}>\{3,6\}>\{4,5\}>\{2,7\}>\{0,9\} .
\end{aligned}
$$

Observe that, in general, the largest element in $T_{n}$ is the pair $\{1, n-1\}$, that is, the pair containing the element 1.

Theorem 5.4 Let $P$ be a palindrome that starts and ends in $p$. Let $k$ be a positive integer such that $k$ is less than $p$. For $\{a, b\},\{c, d\} \in T_{n}$ such that $\{a, b\}>\{c, d\}$, we have

$$
\left(k^{a}, P, k^{b}\right)>\left(k^{c}, P, k^{d}\right) .
$$




\begin{tabular}{l|rrrrr} 
& 0 & 1 & 2 & 3 & 4 \\
\hline 0 & 1 & & & & \\
1 & 3 & & & & \\
2 & 9 & 11 & & & \\
3 & 35 & 40 & & & \\
4 & 155 & 181 & 169 & & \\
5 & 791 & 917 & 875 & & \\
6 & 4529 & 5263 & 4985 & 5095 & \\
7 & 28839 & 33486 & 31794 & 32256 & \\
8 & 201939 & 234541 & 222509 & 226291 & 224621 \\
9 & 1542739 & 1791647 & 1700193 & 1727682 & 1719102
\end{tabular}

Figure 5: Values of $\beta\left(1^{i}, 2,1^{n-i}\right)$ for $2 \cdot i \leq n \leq 9$.

Proof: Substitute $j=0$ in Proposition 5.3 and use Corollary 3.8 with $N=\left(k^{a}\right)$ for suitable values of $a$. The details are left to the reader.

The values of $\beta\left(1^{i}, 2,1^{n-i}\right)$ are presented in Figure 5 . Observe how the values in each row alternate. The values in the odd columns are decreasing, and values in the even columns are increasing. Hence, the largest element is always in the column $i=1$, and the smallest is in the column $i=0$.

Corollary 5.5 Let $P$ be a palindrome that starts and ends in $p$. Let $k$ be a positive integer such that $k$ is less than $p$. Then, among all lists of the form $\left(k^{i}, P, k^{j}\right)$, where $i+j=n$, the two lists $\left(k, P, k^{n-1}\right)$ and $\left(k^{n-1}, P, k\right)$ are the unique maximal elements in the preorder $\geq$.

\section{On the balancing of lists}

The following theorem determines the lists that are maximal in the preorder $\geq$, among all lists of size $n$ and length $d$. It was originally conjectured by Gessel.

Theorem 6.1 Let $n$ and $d$ be positive integers. Let $k=\lfloor n / d\rfloor, a=n-k \cdot d$ and $b=d-a-1$. Then among all lists of size $n$ and length $d$, the two lists $\left(k,(k+1)^{a}, k^{b}\right)$ and $\left(k^{b},(k+1)^{a}, k\right)$ are the largest in the preorder $\geq$.

We call a list $B$ balanced if each entry is $k$ or $k+1$ for some positive integer $k$. As a first step in the proof of Theorem 6.1, we show that for any given list, we can find a balanced list of the same length, which is greater in the preorder $\geq$. The second step is to identify the maximal elements among all the balanced lists of a given length.

Theorem 6.2 For every list $M$, there exists a balanced list $B$ such that $B \geq M$, where the list $B$ has the same size and length as the list $M$. 
This theorem will follow as a special case of Theorem 6.7. We now work towards the proof of Theorem 6.7.

Lemma 6.3 Let $m_{1}, m_{2}, n_{1}, n_{2}$ be non-negative integers such that $m_{1}+n_{1}=m_{2}+n_{2}$ and $\mid m_{1}-$ $n_{1}|\leq| m_{2}-n_{2} \mid$. Let $P$ be a palindrome, where $P$ could possibly be the empty list. Then we have, $\left(m_{1}, P, n_{1}\right) \geq\left(m_{2}, P, n_{2}\right)$ with equality iff $\left|m_{1}-n_{1}\right|=\left|m_{2}-n_{2}\right|$.

Proof: By reversing the lists if necessary, we may assume that $m_{1} \leq n_{1}$ and $m_{2}<n_{2}$. Hence, it is enough to show that $(m, P, n)>(m-1, P, n+1)$, where $m \leq n$. Note that $(m, P, m-1) \geq(m-1, P, m)$, since they are reverse of each other. Applying Lemma 4.3, we get $(m, P, m) \succ(m-1, P, m+1)$. Now, by Lemmas 3.6 and 3.7, we obtain the desired inequality $(m, P, n)>(m-1, P, n+1)$.

If the palindrome $P$ has length 1 , that is, $P$ may be written as $(p)$, then this lemma is equivalent to Proposition 4.1 in [11]. Also, by setting $P$ to be the empty list, we obtain the unimodality of the binomial coefficients.

Lemma 6.4 Let $m_{1}, m_{2}, n_{1}$ and $n_{2}$ be positive integers such that $m_{1}+n_{1}=m_{2}+n_{2}$ and $\left|m_{1}-n_{1}\right|<$ $\left|m_{2}-n_{2}\right|$. Let $P$ be a palindrome and $L$ and $L^{\prime}$ be two lists. Any of the two lists $P$ and $L$ is allowed to be the empty list $\epsilon$, but $L^{\prime}$ must be a non-empty list. Then we have $\left(L, m_{1}, P, n_{1}, L^{\prime}\right) \succ\left(L, m_{2}, P, n_{2}, L^{\prime}\right)$.

Proof: First observe that the statement $\left|m_{1}-n_{1}\right|<\left|m_{2}-n_{2}\right|$ is equivalent to $\left|m_{1}-n_{1}\right|+2 \leq\left|m_{2}-n_{2}\right|$, which implies $\left|m_{1}-\left(n_{1}-1\right)\right| \leq\left|m_{2}-\left(n_{2}-1\right)\right|$. Also, observe that $n_{1} \neq n_{2}$. We now prove the lemma in three cases.

- The list $L$ is empty and $n_{1}<n_{2}$. By Lemma 6.3 , we have $\left(m_{1}, P, n_{1}-1\right) \geq\left(m_{2}, P, n_{2}-1\right)$. Hence by Lemma 4.3 , we obtain $\left(m_{1}, P, n_{1}\right) \succ\left(m_{2}, P, n_{2}\right)$. By Corollary 3.8, we get $\left(m_{1}, P, n_{1}, L^{\prime}\right) \succ$ $\left(m_{2}, P, n_{2}, L^{\prime}\right)$.

- The list $L$ is empty and $n_{1}>n_{2}$. Since $n_{1}>n_{2}$ we have $m_{2}>n_{2}$. Hence $\left|m_{1}-\left(n_{1}-1\right)\right| \leq$ $\left|m_{1}-n_{1}\right|+1<\left|m_{2}-n_{2}\right|+1=\left|m_{2}-\left(n_{2}-1\right)\right|$. That is, the inequality $\left|m_{1}-\left(n_{1}-1\right)\right|<\left|m_{2}-\left(n_{2}-1\right)\right|$ is strict. Hence by Lemma 6.3 , we get the two statements $\left(m_{1}, P, n_{1}-1\right)>\left(m_{2}, P, n_{2}-1\right)$ and $\left(m_{1}, P, n_{1}\right) \geq\left(m_{2}, P, n_{2}\right)$. Now, applying Lemma 4.4 with $k=0$, we obtain $\left(m_{1}, P, n_{1}, 1\right) \succ$ $\left(m_{2}, P, n_{2}, 1\right)$. Finally, by Lemma 3.7 and Corollary 3.8 , we have $\left(m_{1}, P, n_{1}, L^{\prime}\right) \succ\left(m_{2}, P, n_{2}, L^{\prime}\right)$.

- The list $L$ is non-empty. Using the conclusions of the previous two cases, we obtain the comparison $\left(n_{1}, P, m_{1}, L^{*}\right) \succ\left(n_{2}, P, m_{2}, L^{*}\right)$. Consider the weaker preorder and reverse both sides to obtain $\left(L, m_{1}, P, n_{1}\right) \geq\left(L, m_{2}, P, n_{2}\right)$.

By Lemma 6.3, we have $\left(n_{1}-1, P, m_{1}\right) \geq\left(n_{2}-1, P, m_{2}\right)$ and $\left(n_{1}-1, P, m_{1}-1\right)>\left(n_{2}-1, P, m_{2}-1\right)$. Applying Lemma 4.4 to these two inequalities, we obtain $\left(n_{1}-1, P, m_{1}, 1\right) \succ\left(n_{2}-1, P, m_{2}, 1\right)$. Now, by Lemmas 3.6 and 3.7, we get $\left(n_{1}-1, P, m_{1}, L^{*}\right)>\left(n_{2}-1, P, m_{2}, L^{*}\right)$, which by reversing is equivalent to $\left(L, m_{1}, P, n_{1}-1\right)>\left(L, m_{2}, P, n_{2}-1\right)$.

Applying Lemma 4.4 to the last two conclusions, we have $\left(L, m_{1}, P, n_{1}, 1\right) \succ\left(L, m_{2}, P, n_{2}, 1\right)$. Finally, by Lemma 3.7 and Corollary 3.8, we obtain $\left(L, m_{1}, P, n_{1}, L^{\prime}\right) \succ\left(L, m_{2}, P, n_{2}, L^{\prime}\right)$. 
These three cases complete the proof of the lemma.

Corollary 6.5 Let $m_{1}, m_{2}, n_{1}$ and $n_{2}$ be positive integers such that $m_{1}+n_{1}=m_{2}+n_{2}$ and $\left|m_{1}-n_{1}\right|<$ $\left|m_{2}-n_{2}\right|$. Let $P$ be a palindrome and $M$ and $N$ two lists. Any of the three lists $P, M$ and $N$ is allowed to be the empty list $\epsilon$. Then we have $\left(M, m_{1}, P, n_{1}, N\right)>\left(M, m_{2}, P, n_{2}, N\right)$.

Proof: If both $M$ and $N$ are empty, then the result follows by Lemma 6.3. Hence, we may assume that one of the lists $M$ and $N$ is non-empty. If $N$ is non-empty then the result follows by Lemma 6.4. The last case to consider is when $N$ is empty and $M$ is non-empty. By Lemma 6.4, we have $\left(n_{1}, P, m_{1}, M^{*}\right) \succ\left(n_{2}, P, m_{2}, M^{*}\right)$. Now, by considering the weaker preorder and reversing the lists, the result follows.

Proposition 6.6 Let $L, M$ and $L^{\prime}$ be lists such that $L^{\prime}$ is non-empty. Then there exists a balanced list $B$ such that $\left(L, B, L^{\prime}\right) \succeq\left(L, M, L^{\prime}\right)$, where $B$ has the same size and length as $M$.

Proof: Let $d$ be the length of the list $M$. We use induction on $d$. The theorem is clearly true for $d=1$. By Lemma 6.4, the conclusion is true when $d=2$. Assume that the theorem is true for $d-1$. We will now show that it is also true for $d$.

On a list of the form $\left(L, n_{1}, N, n_{2}, L^{\prime}\right)$, where $N$ has length $d-2$, define the following two operations:

- By the induction hypothesis, we can find a balanced list $\left(k_{1}, K\right)$ such that $\left(L, k_{1}, K, n_{2}, L^{\prime}\right) \succeq$ $\left(L, n_{1}, N, n_{2}, L^{\prime}\right)$. Let $\left(L, k_{1}, K, n_{2}, L^{\prime}\right)$ be the result of the first operation.

- By the induction hypothesis, we can find a balanced list $\left(K, k_{2}\right)$ such that $\left(L, n_{1}, K, k_{2}, L^{\prime}\right) \succeq$ $\left(L, n_{1}, N, n_{2}, L^{\prime}\right)$. Let $\left(L, n_{1}, K, k_{2}, L^{\prime}\right)$ be the result of the second operation.

Let $\left(n_{1}, N, n_{2}\right)$ be a list of length $d$. By alternating the above two operations on the original list $\left(L, n_{1}, N, n_{2}, L^{\prime}\right)$, we obtain a list $\left(L, k_{1}, K, k_{2}, L^{\prime}\right)$ such that $\left(k_{1}, K\right)$ and $\left(K, k_{2}\right)$ are both balanced lists. But observe that the list $\left(k_{1}, K, k_{2}\right)$ is not necessarily balanced. If it is balanced, then the proof of the induction step is done, else it has the form $(n+1, n, \ldots, n, n-1)$ or $(n-1, n, \ldots, n, n+1)$. Applying Lemma 6.4 using the palindrome $P=(n, \ldots, n)$, we can balance this list and hence complete the induction.

As an example, consider the list $\left(L, 4,9,5, L^{\prime}\right)$, where $L^{\prime}$ is not the empty list. We can balance the middle part of this list by the following moves:

$$
\left(L, 4,9,5, L^{\prime}\right) \prec\left(L, 4,7,7, L^{\prime}\right) \prec\left(L, 5,6,7, L^{\prime}\right) \prec\left(L, 6,6,6, L^{\prime}\right),
$$

where in the last step we balanced around the palindrome (6). 
Note that Proposition 6.6 fails when $L^{\prime}$ is the empty list. One may construct such an example using Lemma 4.3. Also observe that in Proposition 6.6 (and in Theorem 6.7), the balanced list $B$ only depends on the list $M$ and does not depend on the lists $L$ and $L^{\prime}$.

Theorem 6.7 Let $L, M$ and $L^{\prime}$ be three lists. Then there exists a balanced list $B$ such that $\left(L, B, L^{\prime}\right) \geq$ $\left(L, M, L^{\prime}\right)$, where the list $B$ has the same size and length as $M$.

Proof: Consider the list $\left(L, M, L^{\prime}, 1\right)$. By Proposition 6.6, we can find a balance list $B$ such that $\left(L, B, L^{\prime}, 1\right) \succeq\left(L, M, L^{\prime}, 1\right)$, where $B$ has the same size and length as $M$. This inequality implies that $\left(L, B, L^{\prime}\right) \geq\left(L, M, L^{\prime}\right)$.

Theorem 6.2 follows now by setting $L$ and $L^{\prime}$ both to be the empty list. In order to prove Theorem 6.1, we now consider the problem of rearranging the entries of a list, in order to get higher up in the preorder $\geq$. To do this, we have the following lemma:

Lemma 6.8 Let $k$ be a positive integer and let $P$ be a palindrome that starts (and ends) in an integer greater than $k$. For $i$ and $j$ non-negative integers such that $j>0$, we have

$$
\left(k^{i}, k^{j}, P, k+1\right) \succ\left(k^{i}, P, k^{j}, k+1\right) .
$$

Proof: Assume that $P$ has length at least 2. The case when $P$ has length 1 can be done similarly. Since $P$ is palindromic, we can write $P=(p, Q, p)$, with $p$ larger than $k$ and $Q$ a palindrome. Since $\left(p-1, Q, p, k^{j-1}, k-1\right)$ and $\left(k-1, k^{j-1}, p, Q, p-1\right)$ are reverse of each other, we get $\left(p-1, Q, p, k^{j-1}, k-\right.$ $1) \geq\left(k-1, k^{j-1}, p, Q, p-1\right)$. Applying Lemma 4.3, we obtain $\left(p-1, Q, p, k^{j}\right) \succ\left(k-1, k^{j-1}, p, Q, p\right)$. Now, by Corollary 3.8, we have $\left(p-1, Q, p, k^{j}, k^{i}\right) \succ\left(k-1, k^{j-1}, p, Q, p, k^{i}\right)$. Taking the weaker preorder and reversing, we get $\left(k^{i}, k^{j}, p, Q, p-1\right)>\left(k^{i}, p, Q, p, k^{j-1}, k-1\right)$. Also note that by Corollary 5.5, $\left(k^{i}, k^{j}, p, Q, p, k\right) \geq\left(k^{i}, p, Q, p, k^{j}, k\right)$. Applying Lemma 4.4 to the last two inequalities, we get the conclusion of the lemma.

By our definition, a balanced list consists of two distinct consecutive elements, say $k$ and $k+1$. However, it is more interesting to look at a general situation, where a list is made up of two integers $k$ and $p$, not necessarily consecutive.

Corollary 6.9 Let $a$ and $b$ be non-negative integers and $a>0$. Among all lists that have $a$ 's and $b+1 k$ 's, where $k<p$, the two lists $\left(k, p^{a}, k^{b}\right)$ and $\left(k^{b}, p^{a}, k\right)$ are the maximal elements in the preorder $\geq$.

Proof: Let $L$ be a list that has $a p$ 's and $b+1 k$ 's. In view of Corollary 5.5 , it suffices to get a list $L^{\prime}$ of the form $\left(k^{i}, p^{a}, k^{j}\right)$, for suitable integers $i$ and $j$, such that $L^{\prime} \geq L$. 
Hence, we may assume that $L$ has the form $\left(k^{i}, p^{c}, k^{j}, p^{d}, M\right)$, for positive integers $c, j$ and $d$ and a list $M$. By Lemma 6.8, we have $\left(k^{i}, k^{j}, p^{c}, k+1\right) \succ\left(k^{i}, p^{c}, k^{j}, k+1\right)$. Now, by Lemma 3.7 and Corollary 3.8, we get $\left(k^{i}, k^{j}, p^{c}, p^{d}, M\right) \succ\left(k^{i}, p^{c}, k^{j}, p^{d}, M\right)$. We repeat this argument to obtain a list where all the $p$ 's are collected together. That is, we get $L^{\prime}$ of the form $\left(k^{i}, p^{a}, k^{j}\right)$, for suitable integers $i, j$ and $a$, such that $L^{\prime} \geq L$.

Theorem 6.1 follows now by applying Theorem 6.2 and Corollary 6.9 with $p=k+1$.

\section{An application to inequalities for the Euler numbers}

The following statement is due to MacMahon [7, Article 159]. For $S$ a subset of $\{1, \ldots, n-1\}$ and $T$ a subset of $\{1, \ldots, m-1\}$, we have

$$
\left(\begin{array}{c}
n+m \\
n
\end{array}\right) \cdot \beta(S) \cdot \beta(T)=\beta(S \cup(T+n))+\beta(S \cup\{n\} \cup(T+n)),
$$

where $T+n$ denotes the shifted set $T+n=\{t+n: t \in T\}$. For lists, this statement breaks into two identities. Let $(L, l)$ be a list of size $n-1$ and $(k, K)$ be a list of size $m-1$. Then

$$
\begin{aligned}
\left(\begin{array}{c}
n+m \\
n
\end{array}\right) \cdot \beta(L, l) \cdot \beta(k, K) & =\beta(L, l+1+k, K)+\beta(L, l, 1, k, K) \\
& =\beta(L, l+1, k, K)+\beta(L, l, 1+k, K) .
\end{aligned}
$$

Also observe that $\left(\begin{array}{c}n+1 \\ 1\end{array}\right) \cdot \beta(L, l)=\beta(L, l+1)+\beta(L, l, 1)$.

Now, we are able to prove:

Proposition 7.1 Let $a, b, c, d$ be non-negative integers, such that $a+b=c+d=n$. Then for $\{a, b\}>\{c, d\}$ in the linear order $T_{n}$, we have

$$
\left(\begin{array}{l}
n \\
a
\end{array}\right) \cdot E_{a} \cdot E_{b}>\left(\begin{array}{l}
n \\
c
\end{array}\right) \cdot E_{c} \cdot E_{d} .
$$

Proof: Consider first the case when $a, b, c, d \geq 2$. Then by MacMahon's identity, we have $\left(\begin{array}{l}n \\ a\end{array}\right) \cdot E_{a} \cdot E_{b}=$ $E_{n}+\beta\left(1^{a-2}, 3,1^{b-2}\right)$. Since the inequality $\{a-2, b-2\}>\{c-2, d-2\}$ in the linear order $T_{n-4}$ is equivalent to $\{a, b\}>\{c, d\}$ in $T_{n}$, the result follows, in this case, by Theorem 5.4.

It remains to consider the elements $\{0, n\}$ and $\{1, n\}$ in the linear order $T_{n}$. But $\left(\begin{array}{l}n \\ 0\end{array}\right) \cdot E_{0} \cdot E_{n}=$ $E_{n}<E_{n}+\beta\left(3,1^{n-4}\right)=\left(\begin{array}{c}n \\ 2\end{array}\right) \cdot E_{2} \cdot E_{n-2}$. Hence $\{0, n\}$ corresponds to the smallest element. To obtain that $\{1, n-1\}$ corresponds to the largest element, begin to observe that $\beta\left(2,1^{n-3}\right)>\beta\left(2,2,1^{n-5}\right)>$ $\beta\left(1,3,1^{n-5}\right)$ by Lemma 3.9 and Theorem 6.7. Thus, we have $\left(\begin{array}{l}n \\ 1\end{array}\right) \cdot E_{1} \cdot E_{n-1}=E_{n}+\beta\left(2,1^{n-3}\right)>$ $E_{n}+\beta\left(1,3,1^{n-5}\right)=\left(\begin{array}{l}n \\ 3\end{array}\right) \cdot E_{3} \cdot E_{n-3}$, and the proposition follows. 


\section{Concluding remarks}

It is interesting to note that, for deciding the maximum of $\beta(L)$ for a given class of lists, we have not calculated any values of $\beta(L)$ explicitly. Instead, we have been building comparisons between lists from comparisons of smaller lists. Formulas for computing $\beta(L)$ can be found in $[3,6]$ and $[7$, Article 157].

The order relation $\geq$ is a preorder on the set of lists, and not a partial order since it does not satisfy antisymmetry. Let $L$ and $M$ be two lists of the same size such that $\beta(L)=\beta(M)$. Would this imply that $L=M$ or $L=M^{*}$ ? That would say that the relation $\geq$ is a partial order on the set of pairs $\left\{L, L^{*}\right\}$ of lists.

In a forthcoming paper, we will generalize our results to $p$-augmented $r$-signed permutations; see [5] for their definition. They have been studied for the case when $p=1$ in [4]. Steingrímsson has also studied $r$-signed permutations under the name indexed permutations [13]. When $r=2$ and $p=1$, the $p$-augmented $r$-signed permutations are known as signed permutations. Readdy [10] obtained that when maximizing over signed permutations with 2 , respectively 3 , runs, maximum occurs at the list with the proportions $(1: 2)$, respectively $(1: 2: 2)$. By extending the techniques in this paper, we have obtained a generalization of the Gessel conjecture and Readdy's result for signed permutations: when maximizing the descent statistic over lists of length $d$, the maximum occurs when the lengths of runs have the proportions $(p: \underbrace{r: \cdots: r}_{d-1})$.

For a non-negative integer $d$, let $f(n)$ denote the maximum value of the descent statistic $\beta(L)$, where $L$ ranges over all lists of size $n$ and length $d$. We conjecture that the asymptotic behavior of $f(n)$ is given by

$$
f(n)=\Theta\left(\frac{d^{n}}{n^{(d-1) / 2}}\right) .
$$

This is proved for the two cases $d=2$ and $d=3$ in [11].

There is a $q$-analogue of Lemma 2.4 by refining the statistics with the number of inversions of the permutation. Define linear maps $\alpha_{n}, \beta_{n}: \mathbb{Z}[q]^{n} \longrightarrow \mathbb{Z}[q]^{n+1}$ by

$$
\begin{aligned}
& \alpha_{n}\left(v_{1}, \ldots, v_{n}\right)=\left(0, q^{n-1} \cdot v_{1}, q^{n-2} \cdot\left(v_{1}+v_{2}\right), \ldots, q \cdot\left(v_{1}+\cdots+v_{n-1}\right), v_{1}+\cdots+v_{n}\right), \\
& \beta_{n}\left(v_{1}, \ldots, v_{n}\right)=\left(q^{n} \cdot\left(v_{1}+\cdots+v_{n}\right), q^{n-1} \cdot\left(v_{2}+\cdots+v_{n}\right), \ldots, q^{2} \cdot\left(v_{n-1}+v_{n}\right), q \cdot v_{n}, 0\right) .
\end{aligned}
$$

For a subset $S$ of $\{1, \ldots, n\}$, consider the vector $\left(w_{1}, \ldots, w_{n+1}\right)=\gamma_{n}\left(\cdots \gamma_{1}(1) \cdots\right)$, where $\gamma_{i}=\beta_{i}$ if $i \in S$, otherwise $\gamma_{i}=\alpha_{i}$. Then $w_{i}$ is given by the sum $\sum_{\pi} q^{\operatorname{inv}(\pi)}$ where $\pi$ ranges over all permutations in the symmetric group $\mathcal{S}_{n+1}$ with descent set $S$ and $\pi_{n+1}=i$.

\section{Acknowledgments}

We thank Ira Gessel, who told us about his conjecture. We also thank Richard Stanley, who directed us to essential references. 


\section{References}

[1] A. BJörner, Shellable and Cohen-Macaulay partially ordered sets, Trans. Amer. Math. Soc. 260 (1980), 159-183. 3

[2] N. G. De Bruijn, Permutations with given ups and downs, Nieuw Arch. Wisk 18 (1970), 61-65. 2

[3] L. Carlitz, Permutations with prescribed pattern, Math. Nachr. 58 (1973), 31-53. 20

[4] R. Ehrenborg and M. Readdy, The r-cubical lattice and a generalization of the cd-index, European J. Combin. 17 (1996), 709-725. 2, 10, 20

[5] R. Ehrenborg and M. Readdy, Sheffer posets and $r$-signed permutations, Ann. Sci. Math Québec 19 (1995), 173-196. 20

[6] H. O. Foulkes, Enumeration of permutations with prescribed up-down and inversion sequences, Discrete Math 15 (1976), 235-252. 20

[7] P. A. MacMahon, "Combinatory Analysis, Vol. I," Chelsea Publishing Company, New York, 1960. 19, 20

[8] J. Millar, N. J. A. Sloane and N. E. Young, A new operation on sequences: The boustrophedon transform, J. Combin. Theory Ser. A 76 (1996), 44-54. 3, 4, 5

[9] I. Niven, A combinatorial problem on finite sequences, Nieuw Arch. Wisk. 16 (1968), 116-123. 2

[10] M. Readdy, Extremal problems in the face lattice of the $n$-dimensional octahedron, Discrete Math 139 (1995), 361-380. 2, 20

[11] B. E. Sagan, Y.-N. Yeh and G. Ziegler, Maximizing Möbius functions on subsets of Boolean algebras, Discrete Math 126 (1994), 293-311. 2, 16, 20

[12] R. P. Stanley, "Enumerative Combinatorics, Vol. I," Wadsworth and Brooks/Cole, Pacific Grove, 1986. 3

[13] E. Steingrímsson, Permutation statistics of indexed permutations, European J. Combin. 15 (1994), 187-205. 20

[14] G. Viennot, Permutations ayant une forme donnée, Discrete Math 26 (1979), 279-284. 2, 5, 10

[15] G. Viennot, Équidistribution des permutations ayant une forme donnée selon les avances et coavances, J. Combin. Theory Ser. A 31 (1981), 43-55.

\author{
R. EHRENBorG \\ Department of Mathematics \\ White Hall \\ Cornell University \\ ITHACA, NY 14853-7901 \\ jrge@math.cornell.edu
}

\author{
S. MAHAJAN \\ Department of Mathematics \\ White Hall \\ Cornell University \\ ITHACA, NY 14853-7901 \\ swapneel@math.cornell.edu
}

